\title{
Pulse phase and precession phase resolved spectroscopy of Hercules X-1: studying a representative Main-On with RXTE
}

\author{
D. Vasco ${ }^{1}$, R. Staubert ${ }^{1}$, D. Klochkov ${ }^{1}$, A. Santangelo ${ }^{1}$, N. Shakura ${ }^{2}$, and K. Postnov ${ }^{2}$ \\ ${ }^{1}$ Institut für Astronomie und Astrophysik, Universität Tübingen, Sand 1, 72076 Tübingen, Germany \\ e-mail: vasco@astro.uni-tuebingen.de \\ 2 Sternberg Astronomical Institute, Lomonossov University Moscow, Russia \\ Received 7 August 2012 / Accepted 22 December 2012
}

\section{ABSTRACT}

\begin{abstract}
Aims. We performed a detailed pulse phase resolved spectroscopy of the accreting binary X-ray pulsar Her X-1 in the energy range 3.5-75 keV and have established pulse phase profiles for all spectral parameters. For three parameters, the centroid energy of the cyclotron line, the photon index and the flux of the $6.4 \mathrm{keV}$ iron line, we have studied the variation as a function of $35 \mathrm{~d}$ phase.

Methods. We analyzed RXTE observations of the Main-On of November 2002 using data from the PCA and the HEXTE instruments. Four different time intervals of about $\sim 1 \mathrm{~d}$ duration were selected to provide a good coverage of a complete Main-On. The intervals are centered at $35 \mathrm{~d}$ phases $0.03,0.10,0.15$, and 0.20 , respectively.

Results. All spectral parameters show a strong modulation with pulse phase. While the centroid energy of the cyclotron line follows roughly the shape of the pulse profile, showing higher values close to the peak of the X-ray pulse, both the photon index and the iron line intensity exhibit distinct minima around the peak of the X-ray pulse. With respect to variations of the observed profiles with $35 \mathrm{~d}$ phase, we find that there is a clear evolution of the shape of the pulse profiles (flux versus pulse phase), a moderate increase of the maximum cyclotron line energy (found around pulse phase 0.7 ), but no significant evolution of the shape of the pulse phase profiles of the cyclotron line energy, the spectral power law index or the iron line intensity.

Conclusions. The variation of spectral parameters as a function of the pulse phase provides important information about the system: $i$. the disappearance of the Fe line flux near the highest continuum flux may be an indication of a hollow cone geometry of the accretion structure; ii. the apparent non-dependence of the cyclotron line energy profiles on $35 \mathrm{~d}$ phase provides a new possibility to test the model of free precession of the neutron star, proposed to be responsible for the systematic variations in the pulse profiles.
\end{abstract}

Key words. pulsars: individual: Her X-1 - stars: neutron - binaries: general

\section{Introduction}

The X-ray source Her X-1 was discovered in 1972 by Uhuru (Tananbaum et al. 1972), and classified as an accreting X-ray binary. Her X-1 is one of the brightest and most studied persistent binary X-ray pulsars. The distance to the system is $\sim 7 \mathrm{kpc}$ and the masses of the neutron star and its companion are approximately $1.5 M_{\odot}$ and $2.2 M_{\odot}$, respectively (Reynolds et al. 1997). The X-ray flux shows periodic modulation on several different time-scales: pulsations with $1.24 \mathrm{~s}$ due to the spin of the neutron star, eclipses due to the $1.7 \mathrm{~d}$ orbital period, and a $35 \mathrm{~d}$ super-orbital period due to obscuration of the X-ray emitting region by the precessing accretion disk. This $35 \mathrm{~d}$ periodicity shows different states: two On-states with the $\sim 11 \mathrm{~d}$ Main-On (at phase 0-0.31) and the $\sim 5 \mathrm{~d}$ Short-On (at phase 0.57-0.79), separated by two Off-states (see e.g. Giacconi et al. 1973; Gerend \& Boynton 1976; Boynton et al. 1980; Scott \& Leahy 1999; Scott et al. 2000; Klochkov et al. 2006). The accretion disk is thought to be tilted (with respect to the orbital plane), warped and counter-precessing with a somewhat variable period of $\sim 35 \mathrm{~d}$. The onset of the flux (often identified with $35 \mathrm{~d}$ phase 0.0 ) is called the Turn-On (TO) and corresponds to the transition from the Off-state to the Main-On. At this time, the outer rim of the disk clears the view to the X-ray emitting region close to the polar caps on the surface of the neutron star, leading to an increase in flux. Vice versa, the decline of the flux towards the end of the Main-On is identified with the inner edge of the accretion disk blocking our view to the X-ray emitting regions. A similar cycle is responsible for the Short-On with a maximum flux of roughly one third that of the Main-On.

Her X-1 was also the first accreting X-ray pulsar for which a cyclotron line in the X-ray spectrum has been discovered (Trümper et al. 1978). This absorption-like feature, now referred to as cyclotron resonance scattering feature (CRSF), is observed around $40 \mathrm{keV}$ and allows to estimate the neutron star's magnetic field. Applying the formula $B_{12}=(1+z) E_{\text {cyc }} / 11.6 \mathrm{keV}$ (where $B_{12}$ is the magnetic field strength in units of $10^{12}$ Gauss, $z$ is the gravitational redshift and $E_{\text {cyc }}$ is the centroid energy of the cyclotron line), the first direct measurement of the magnetic field of a neutron star was achieved $\left(\sim 3 \times 10^{12}\right.$ Gauss for Her X-1). The source shows a positive correlation between the centroid energy of the cyclotron line and the bolometric luminosity (Staubert et al. 2007; Vasco et al. 2011).

Another observational feature of Her X-1 is the evolution of the pulse profile as function of energy and time, or better: $35 \mathrm{~d}$ phase. For both the Main-On and the Short-On, a systematic variation in the shape of the pulse profiles is found. These changes consist of the disappearing of some features in the pulse profile with an increase in energy and/or time. These variations repeat systematically on a time scale of $\sim 35 \mathrm{~d}$. These changes have been well known for some time (see e.g. Deeter et al. 1998; Scott et al. 2000; Trümper et al. 1986). Recently, Staubert et al. $(2010,2013)$ on the basis of RXTE observations, gave a 
Table 1. Summary of the four intervals of the Main-On of $35 \mathrm{~d}$ cycle no. 323 .

\begin{tabular}{lcccc}
\hline \hline Interval & $\begin{array}{c}\text { Limits } \\
\text { MJD }\end{array}$ & $\begin{array}{c}\text { Exposure } \\
{[\mathrm{ks}]}\end{array}$ & $\begin{array}{c}\text { Limits } \\
\text { 35 d phase }\end{array}$ & $\begin{array}{c}\text { Center } \\
\text { 35 d phase }\end{array}$ \\
\hline 1 & $52595.060-52596.743$ & 25 & $0.007-0.056$ & 0.03 \\
2 & $52597.959-52598.719$ & 25 & $0.090-0.112$ & 0.10 \\
3 & $52599.603-52600.726$ & 25 & $0.137-0.161$ & 0.15 \\
4 & $52601.316-52602.406$ & 28 & $0.186-0.217$ & 0.20 \\
\hline
\end{tabular}

Notes. The columns are: the number of the interval, the time interval in MJD, the exposure time, the corresponding $35 \mathrm{~d}$ phase interval, and the center of the $35 \mathrm{~d}$ phase interval.

quantitative description of these changes by providing a template, which can be used to predict the shape of the profile in the 9-13 keV energy range for any $35 \mathrm{~d}$ phase during the Main-On. An extension of this work for other energy ranges and including the Short-On is in preparation.

Trümper et al. (1986), on the basis of observations by Exosat, had proposed that free precession of the neutron star is responsible for those pulse profile changes, due to the variation of the angle of the line of sight to the X-ray emitting regions on the surface of the neutron star. For Her X-1, the dependence of the spectral parameters on pulse phase have been observed by several missions. Early examples are Pravdo et al. (1978) with OSO 8, Pravdo et al. (1979) and Soong et al. (1990) with the A-4 experiment of HEAO-1 and McCray et al. (1982) with HEAO 2 the Einstein Observatory, Voges et al. (1982) with the MPE/AIT ballon experiment, Kahabka (1987) with Exosat, Kunz et al. (1996) with Mir-HEXE. Later examples are Endo et al. (2000) with ASCA, Zane \& Ramsay (2001) with XMM-Newton, Lutovinov et al. (2000) with the ART-P telescope on board of GRANADA and Klochkov et al. (2008) with INTEGRAL.

In this work we present a deep investigation of the variation of key spectral parameters as a function of pulse phase with the finest resolution so far and, for the first time, a discussion of a possible evolution with $35 \mathrm{~d}$ phase. Changes of the spectral parameters with pulse phase are quite common among accreting X-ray pulsars and are generally attributed to a change in the viewing angle of the accretion region (see e.g., Kreykenbohm et al. 2004 and references therein). Here, we focus on the variation of the centroid energy $E_{\text {cyc }}$ of the CRSF around $40 \mathrm{keV}$, the photon index $\Gamma$ and the iron line characteristics. We also address the question whether our data support the free precession model which has been suggested to explain the variation in shape of the pulse profile.

\section{Observations and data analysis}

To carry out this analysis we used observations of Her X-1 of the Rossi X-ray Timing Explorer (RXTE) performed in November 2002. These observations correspond to cycle number 323 (cycle numbering according to Staubert et al. 2009b) centered at MJD 52599.32 with an observed Turn-On of MJD 52594.80. The data of this cycle provide the best coverage of any Main-On of Her X-1 observed with RXTE. Within this cycle, we sum up observations from four selected time intervals at different $35 \mathrm{~d}$ phases in order to have a good coverage of an entire Main-On, avoiding dips and eclipses. All intervals have a duration of about one day (details are given in Table 1). Figure 1 shows the four intervals selected for this analysis.

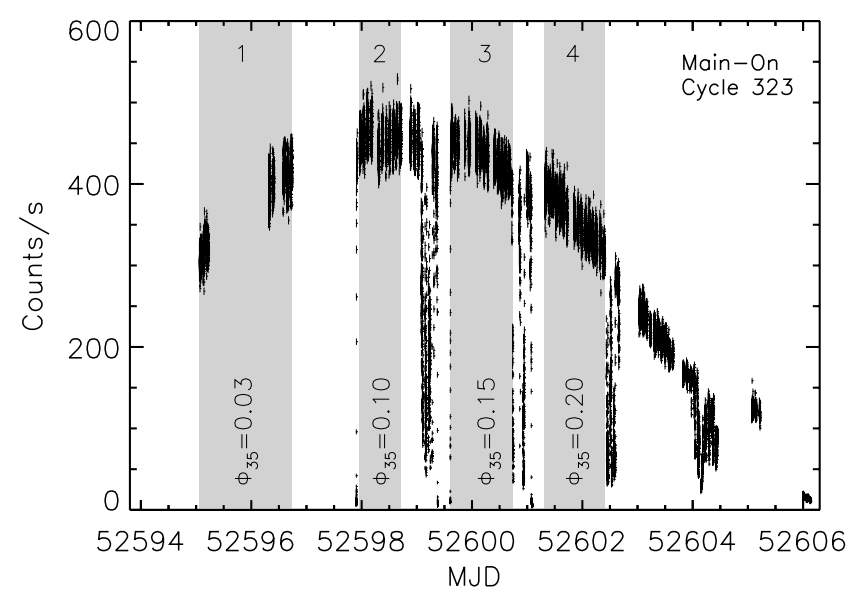

Fig. 1. PCA light curve of the Main-On of cycle 323. The grey zones represent the four intervals used for this analysis. For each interval the centered $35 \mathrm{~d}$ phase is given. Details of the time and $35 \mathrm{~d}$ phase intervals are listed in Table 1.

For barycentric and binary corrections orbital parameters and the ephemeris of Staubert et al. (2009) were used: $P_{\text {orb }}=$ $1.700167287 \mathrm{~d}, \dot{P}_{\text {orb }}=+(2.8 \pm 0.2) \times 10^{-12} \mathrm{~s} \mathrm{~s}^{-1}, a \sin i=13.1831$ lt $-\mathrm{s}$ and $T_{\frac{\pi}{2}}=52599.486440 \mathrm{MJD}$. To align the pulse profiles, a reference time $t_{0}=$ MJD 52594.869900091 and a spin period $P_{\text {spin }}=1.237761809$ s were used. As "pulse phase zero" the sharp-edge feature at the trailing edge of the right shoulder of the main peak was used (see Staubert et al. 2009).

The PCA was used in the energy range $3.5-60 \mathrm{keV}$ and HEXTE in the energy range $20-75 \mathrm{keV}$. The data have been analyzed with XSPEC 12.6.0 ${ }^{1}$ using the highecut ${ }^{2}$ spectral model, power law continuum with an exponential cut-off. The differential photon flux $f(E)$ is described by:

$f(E)=A \begin{cases}E^{-\Gamma} & , \text { if } E \leq E_{\text {cut }} \\ E^{-\Gamma} \exp \left(\frac{E-E_{\text {cut }}}{E_{\text {fold }}}\right), & \text { if } E>E_{\text {cut }}\end{cases}$

where $\Gamma$ is the photon index, $E_{\text {cut }}$ is the cut-off energy and $E_{\text {fold }}$ is the e-folding energy. In addition, two line features are included in the model: a multiplicative Gaussian optical depth for the $\mathrm{CRSF}$ and an additive Gaussian emission line for the iron fluorescence feature around $6.5 \mathrm{keV}$. This model is the same one as used in earlier analyses (see e.g., Coburn et al. 2002 and Staubert et al. 2007). We have consistently found that the highecut spectral model describes the spectrum of Her X-1 best. The discontinuity of this model at the cut-off energy does not constitute a problem and we have verified that no systematic uncertainties are introduced in estimating the spectral parameters. We used the PCA data up to $60 \mathrm{keV}$, which has become feasible by new response matrices (PCA response v11.7, 2009 May 11). These high energy data can definitively improve the photon statistics at energies around and beyond the cyclotron line feature. The same choice has been used in previous analyses (see e.g. Ferrigno et al. 2011) and it was confirmed by Rothschild et al. (2011) with RXTE observations of Cen A. The recorn ${ }^{3}$ spectral component was also added to the model to normalize the background. After we had verified, that the energy and width of the iron $\mathrm{K}$ line is constant $(6.45 \mathrm{keV}$ and $0.5 \mathrm{keV}$, respectively), we fixed

\footnotetext{
1 http://heasarc.gsfc.nasa.gov/docs/xanadu/xspec

2 http://heasarc.gsfc.nasa.gov/xanadu/xspec/manual/ XSmodelHighecut.html

3 http://heasarc.gsfc.nasa.gov/xanadu/xspec/manual/ XSmodelRecorn.html
} 
those values in order to minimize the number of free parameters. GoodXenon ${ }^{4}$ observational modes of the PCA for high resolution analysis were used to exctract light curves and spectra. An additional systematic uncertainty of $1 \%$ was added in using the PCA data. To analyze the data, version v6.11 of the HEASOFT software $^{5}$ and background estimation files Sky_VLE dated 2005 November 28 were used. We have investigated whether there are correlations between the fit parameters: no dependence of any single parameter on the others has been found. All spectral results stated below have been obtained through spectral fits with the assumption of negligible absorption due to neutral hydrogen, both intrinsic to the source and interstellar (which is at $5.1 \times 10^{19} \mathrm{~cm}^{-2}$, Dal Fiume et al. 1998). The introduction of absorption as a free parameter does not lead to a reduction in $\chi^{2}$.

For the pulse phase resolved analysis, we have extracted the spectra in 14 pulse phase bins over the $1.24 \mathrm{~s}$ pulse, the widths of which were chosen to have roughly identical photonstatistics in each spectrum. The centers of the 14 phase bins are at pulse phases: $0.1,0.3,0.45,0.55,0.625,0.675,0.7125$, $0.7375,0.7625,0.7875,0.8250,0.8750,0.9250$, and 0.9750 . For the cyclotron line energy $E_{\text {cyc }}$ this is the finest resolution in pulse phase ever achieved (the four smallest bins around the peak of the pulse have a width of $1 / 80$ of a phase).

With the spectral function described above, good fits with reduced $\chi^{2}$ between 0.9 and 1.2 are generally achieved. However, splitting up the data into small pulse phase intervals reveals that larger $\chi^{2}$ values appear: up to $\sim 12$ for the pulse phase range $0.775-0.85$, and $1.4-2.8$ for the pulse phase range $0.5-0.775$. The main reason for this is the existence of the so called " $10 \mathrm{keV}$ feature" (see the discussion in 3.5). Modeling this feature by an extra Gaussian is generally successful and brings the $\chi^{2}$ down to acceptable values (in a few cases $\chi^{2}$ is still found around 1.3, which is most likely due to an imperfect modeling by a simple Gaussian).

\section{Pulse phase variability}

We concentrate on the detailed study of the variation of the following spectral parameters as function of pulse phase: the centroid energy $E_{\text {cyc }}$ of the cyclotron line, the photon index $\Gamma$, and the intensity of the iron line at $6.4 \mathrm{keV}$. The phase profiles of these spectral parameters are compared with the shape of the flux profiles (the "pulse profiles") for the four different $35 \mathrm{~d}$ phase intervals. In Fig. 2 the $9-13 \mathrm{keV}$ pulse profiles of the four intervals are shown. Note that the pulse profiles are of such high statistical quality that the uncertainties of the flux values (in each of the 128 pulse phase bins) are comparable to the width of the lines chosen to plot the profiles. The profiles are repeated in the following figures (as dashed lines) to allow a direct comparison with the profiles of the spectral parameters. For the remaining spectral parameters, such as the cut-off energy $E_{\text {cut }}$, the folding energy $E_{\text {fold }}$ (with reference to the used spectral model highecut), and the width and depth of the cyclotron line ( $\sigma_{\text {cyc }}$ and $\tau_{\text {cyc }}$ ), we will restrict ourselves to profiles of the combined data of cycle 323. For these parameters the statistics is such, that no statements can be made about a dependence on $35 \mathrm{~d}$ phase. In all Figures, the uncertainties given are one sigma (68\%) values.

\footnotetext{
4 http://heasarc.gsfc.nasa.gov/docs/xte/abc/

pca_issues.html\#configs_modes

5 http://heasarc.gsfc.nasa.gov/ftools/
}

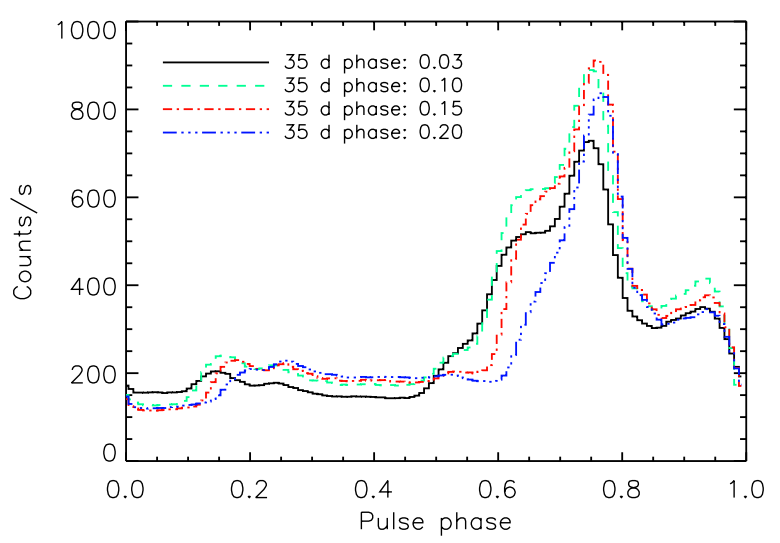

Fig. 2. Mean 9-13 keV pulse profiles of the four time intervals at different $35 \mathrm{~d}$ phases (see Table 1): Interval 1 in black (solid line), Interval 2 in green (dashed line), Interval 3 in red (dash dotted line) and Interval 4 in blue (dash dot dot dotted line). For general $35 \mathrm{~d}$ dependence see Staubert et al. (2010). As timing reference for pulse phase zero we use the "sharp-edge" at the trailing edge of the right shoulder of the main pulse which leads into an eclipse like minimum (Staubert et al. 2009b). Note that these pulse profiles are statistically so accurate that the uncertainties are smaller than the line width.

\subsection{Cyclotron line energy}

The profiles of the cyclotron line energy $E_{\text {cyc }}$ as a function of pulse phase are shown in Figs. 3 and 4 . Generally, $E_{\text {cyc }}$ roughly follows the shape of the pulse profile: the broad maximum is found to be close to the peak of the pulse profile (around pulse phase 0.7 ) for all $35 \mathrm{~d}$ phases. The formal uncertainties of $E_{\text {cyc }}$ (as determined by XSPEC) are on the order of a few to several percent for pulse phases $0.0-0.6$ and $0.9-1.0$ and around one percent or less for pulse phases 0.6-0.9 (close to the peak of the pulse). Adopting an additional systematic uncertainty of $0.15 \mathrm{keV}(\sim 0.4 \%)$ for all data points, the profiles are well represented by cosine functions (see below). We find slightly different values for the mean $E_{\text {cyc }}$ and the peak-to-peak amplitude: a slight, but significant increase is found as function of $35 \mathrm{~d}$ phase. The weighted mean values of the four highest values of $E_{\text {cyc }}$ of each group are $40.16 \pm 0.22 \mathrm{keV}, 40.89 \pm 0.20 \mathrm{keV}$, $41.02 \pm 0.15 \mathrm{keV}$ and $41.52 \pm 0.20 \mathrm{keV}$ for mean $35 \mathrm{~d}$ phases $0.03,0.10,0.15$ and 0.20 (intervals 1 through 4 ), respectively. This corresponds to an increase by $\sim 0.7 \mathrm{keV}$ per 0.1 units in $35 \mathrm{~d}$ phase. The minimum $E_{\text {cyc }}$ values have larger uncertainties with a tendency to decrease with $35 \mathrm{~d}$ phase, but, within uncertainties, they are consistent with a constant value (around $33.3 \mathrm{keV}$ ). The peak-to-peak amplitude is then slightly increasing with $35 \mathrm{~d}$ phase (with a mean of $\sim 7.6 \mathrm{keV}$ ).

While the values for the maximum $E_{\text {cyc }}$ and the peak-to-peak amplitude are dependent on $35 \mathrm{~d}$ phase, the sinusoidal shape of the modulation does not change. To verify this statement, the following analysis was performed: the original $E_{\text {cyc }}$ profiles (Fig. 4) were scaled such that the separation of minimum to maximum $E_{\text {cyc }}$ had a constant value of $7.64 \mathrm{keV}$, using the minimum values measured in phase bin $0.2-0.4$ and the weighted mean of the four highest values in each of the four profiles as reference. In this way, normalized $E_{\text {cyc }}$ profiles were generated. These normalized profiles are shown in Fig. 5, each in comparison to the weighted mean values and a smooth best fit function of the weighted mean: a combination of two cosine functions with common mean $(37.31 \mathrm{keV})$, amplitude $(3.82 \mathrm{keV})$, period and phase-zero values of 0.70 and 0.772 for pulse phases $0.3-0.875$ and 1.47 and 0.3 for the remaining phases, respectively. Visually, there is no obvious deviation of any of the four data sets from 

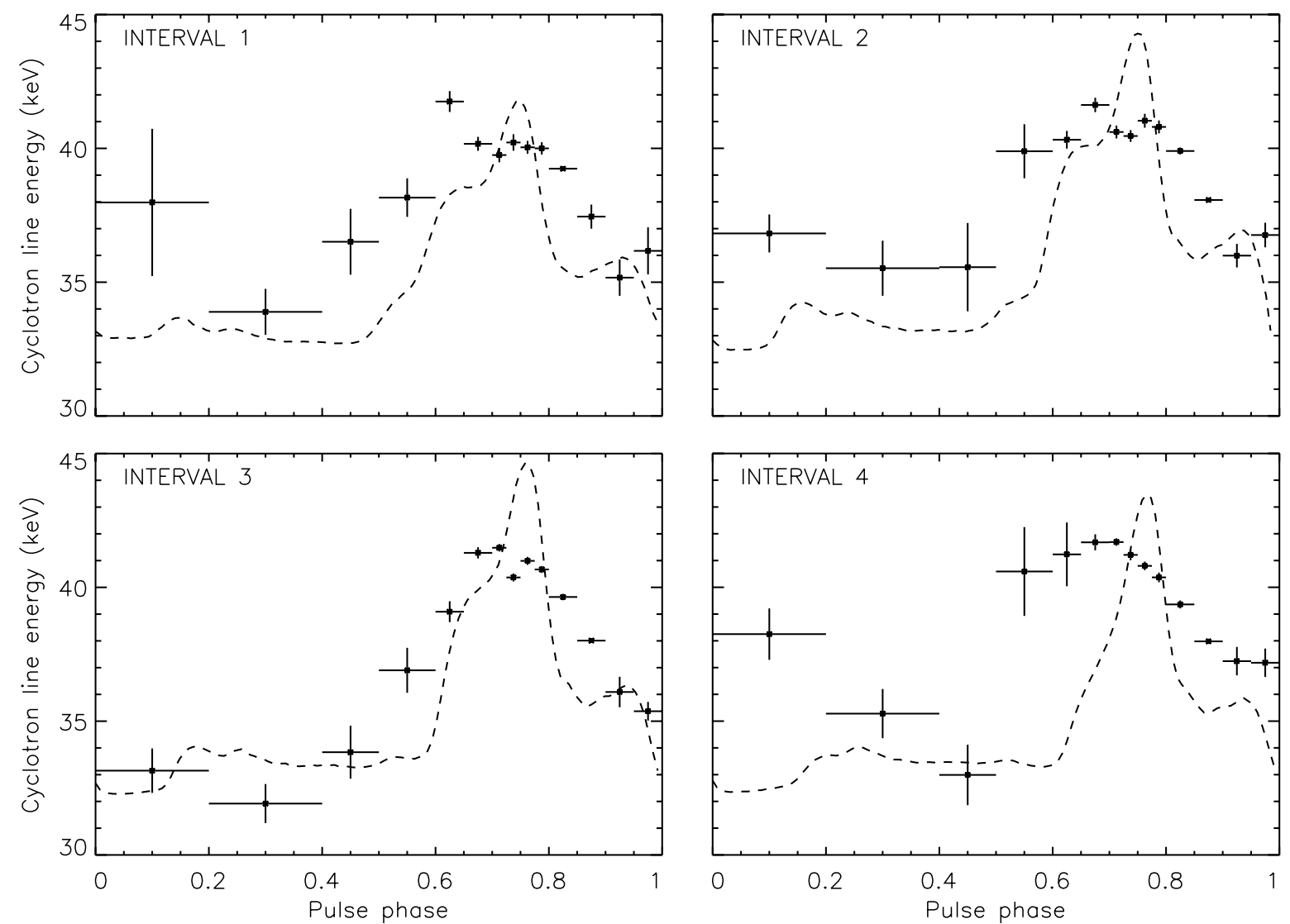

Fig. 3. Cyclotron line centroid energy profiles as function of the pulse phase for Interval 1 (top left), Interval 2 (top right), Interval 3 (bottom left) and Interval 4 (bottom right).

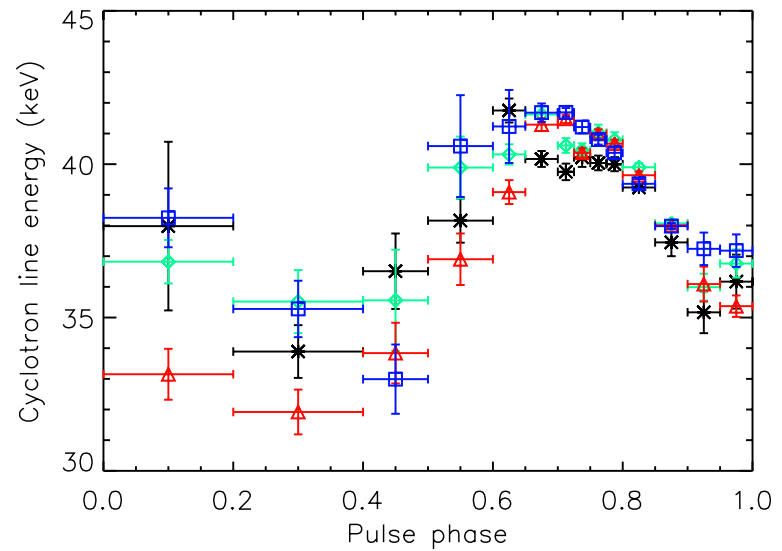

Fig. 4. Cyclotron line centroid energy as function of pulse phase for the four different $35 \mathrm{~d}$ phase intervals (see Table 1): Interval 1 in black (asterisks), Interval 2 in green (diamonds), Interval 3 in red (triangles) and Interval 4 in blue (squares).

the weighted mean. As quantitative statistical test we performed the following: comparing the individual normalized profiles with that profile representing the weighted mean leads to $\chi^{2}$ values (normalized to the degrees of freedom) of $0.72,1.46,0.72$ and 1.32 for the four intervals, respectively. As an additional test, we have then performed a Monte Carlo simulation, in order to answer the following question. Assuming the profiles are indeed the same for all four intervals, that is equal to the best fit mean profile: what is the probability to find an individual profile which deviates from the mean profile in such a way that the $\chi^{2}$ reaches or exceeds the observed value? We have simulated 100 profiles for each of the four intervals, reproducing the statistical conditions of each interval (that is, the widths of the Gaussian distribution from which the random numbers were drawn were chosen according to the uncertainty of the corresponding measured data point). Those probabilities are $68 \%, 29 \%, 73 \%$ and $4 \%$ for the four intervals, respectively. Even $4 \%$ for Interval 4 is a rather high and acceptable probability. We conclude, that, within our statistical accuracy, we find no evidence for any change in the shape of the $E_{\text {cyc }}$ profiles with 35 d phase.

\subsection{Photon-index}

Figure 6 shows the profiles of the photon index $\Gamma$ as function of the pulse phase. This spectral parameter is relatively constant for pulse phases up to 0.6 (showing a small positive slope), but has a distinct dip around the pulse peak and a smaller one around the left shoulder. The photon-indeces range between $0.42 \pm 0.01$ and $1.07 \pm 0.01$ for Interval 1 , between $0.54 \pm 0.01$ and $1.08 \pm 0.02$ for Interval 2, between $0.36 \pm 0.01$ and $1.10 \pm 0.49$ for Interval 3 and between $0.33 \pm 0.01$ and $1.07 \pm 0.01$ for Interval 4 . In general, the spectrum gets harder when the flux increases. While the main features of the pulse phase dependence of $\Gamma$ are very much the same in the four $35 \mathrm{~d}$ intervals, the depth of the new narrow dip around phase 0.62 shows a clear trend towards smaller values from Interval 1 to Interval 4 (see Fig. 7).

\subsection{Iron line intensity}

Another spectral parameter which shows a clear modulation as function of pulse phase is the iron line intensity (see Fig. 8). 

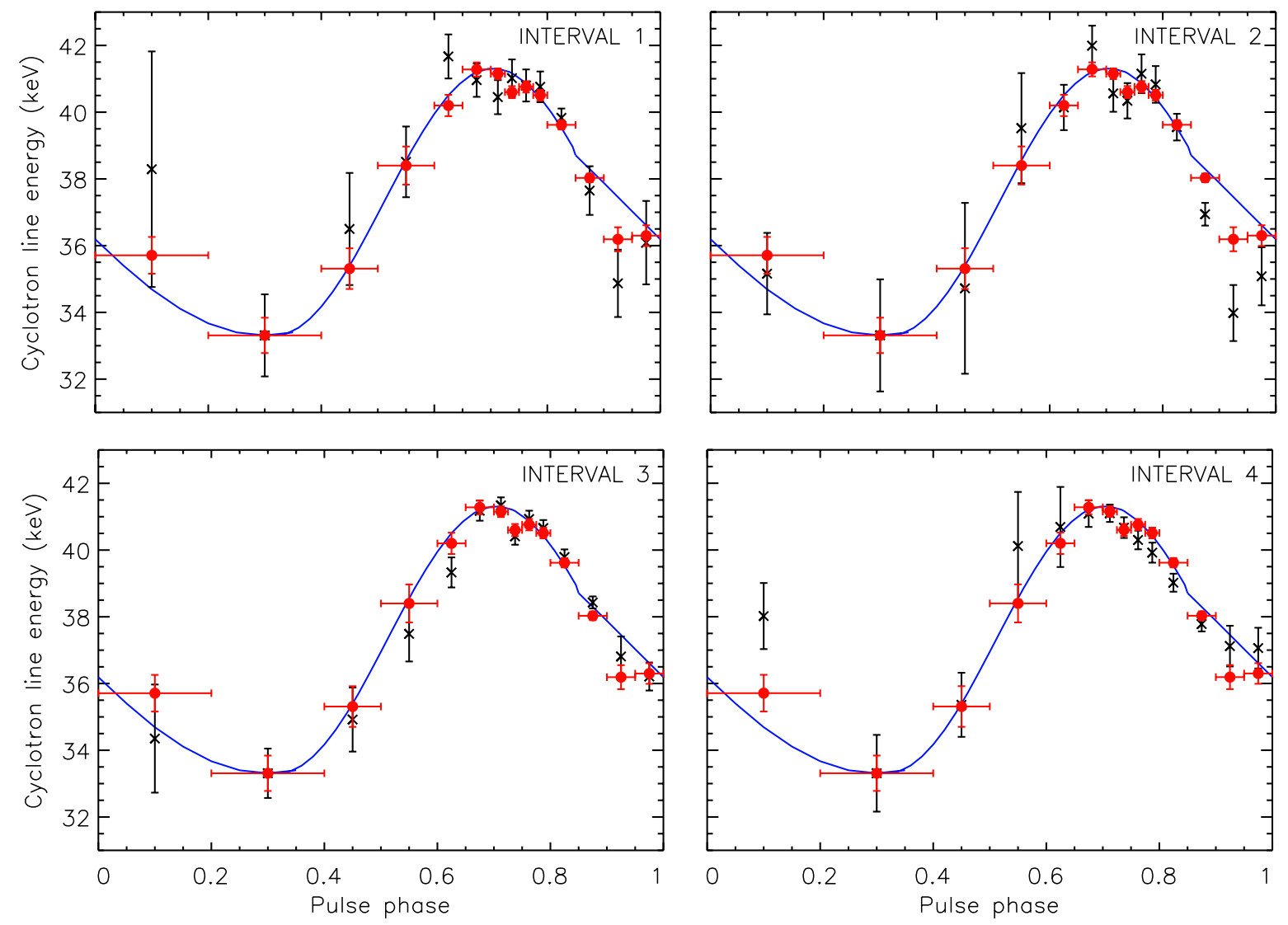

Fig. 5. Cyclotron line profiles of Fig. 3 for the four 35 d intervals (black crosses): Interval 1 (top left), Interval 2 (top right), Interval 3 (bottom left) and Interval 4 (bottom right), normalized to a common amplitude. The big red filled dots represent the weighted mean values of all four intervals. The solid blue line represents a best fit function (a combination of two cosine components). We conclude that, within the given statistics, there is no evidence for any change in shape of the profiles with $35 \mathrm{~d}$ phase.

It shows a moderate increase until the left shoulder of the pulse (phase $\sim 0.7$ ), after which it drops almost to zero around pulse phase 0.8 , before reaching the start value again at pulse phase 0.9. The minimum is coincident with the peak of the X-ray pulse (see Figs. 8 and 9) in a similar way as the photon index. The iron line intensity ranges between 0 (for all intervals) and 12.40 for Interval 1, 14.62 for Interval 2, 13.19 for Interval 3 and 10.75 for Interval 4 . The profiles of $35 \mathrm{~d}$ intervals $2-4$ are quite similar to one another, while for Interval 1 , the iron line intensity is significantly lower for pulse phases $<0.6$.

\subsection{Other spectral parameters}

For the other spectral parameters $E_{\text {cut }}, E_{\text {fold }}$ the width $\sigma_{\text {cyc }}$ and the depth $\tau_{\text {cyc }}$, we show their pulse phase dependence only for the total data sets (not resolved in $35 \mathrm{~d}$ phase), because in these parameters we find a considerably larger scatter, such that no conclusions can be drawn about a possible variation (or not) with $35 \mathrm{~d}$ phase (the $35 \mathrm{~d}$ phase resolved profiles can be found in Vasco 2012) ${ }^{6}$. The profiles are shown in Fig. 10. Also for these parameters there is significant modulation with pulse phase, and they generally show a broad maximum around the phase of the pulse peak, with the interesting feature that for $E_{\text {fold }}$ there is a dip in this maximum, right at the pulse peak. It is also worth noting, that, except for $E_{\text {fold }}$, the measured values for pulse phases $<0.4$ are rather high, not following the shape of the pulse profiles.

\footnotetext{
6 http://tobias-lib.uni-tuebingen.de/frontdoor.php? source_opus $=6346$
}

\subsection{The "10 keV feature"}

It has been noticed (e.g. Coburn et al. 2002), that in fitting observed spectra of accreting X-ray binaries with a cut-off power law a "wiggle" or "bump" around $\sim 10 \mathrm{keV}$ to $\sim 15 \mathrm{keV}$ is found in the residuals. This applies to many different sources including Her X-1 (also to non-cyclotron line sources), to observations with different satellites (e.g. Ginga, BeppoSAX and RXTE), and to analysis with different spectral functions involving a power law (e.g., highecut, plcut, Fermi-Dirac or NPEX). The feature is therefore considered to be inherent to accreting X-ray binaries. So far, no physical explanation has been suggested.

In our current analysis, using data from observations of Her X-1 by RXTE, this feature does not play a role in the phase averaged analysis. However, in the pulse phase resolved analysis, larger $\chi^{2}$ values appear due to the existence of this " $10 \mathrm{keV}$ feature": up to $\sim 12$ for the pulse phase range $0.775-0.85$, and $1.4-2.8$ for the pulse phase range $0.5-0.775$. Modeling this feature by an extra Gaussian is generally successful and brings the $\chi^{2}$ down to acceptable values (in a few cases $\chi^{2}$ is still found around 1.3 , which is most likely due to an imperfect modeling by a simple Gaussian). The centroid energy is generally found at $\sim 16 \mathrm{keV}$ with a $\sigma$ of $\sim 5 \mathrm{keV}$. Figure 11 shows an example of the spectrum of Interval 3, pulse phase bin 0.80-0.85, where the $\chi^{2}$ is reduced from 5.55 to 1.38 for 224 dof. We have verified, that all the other spectral parameters are not altered when introducing this extra Gaussian. We note that this feature occurs most strongly at a pulse phase close to the peak of the pulse, where 

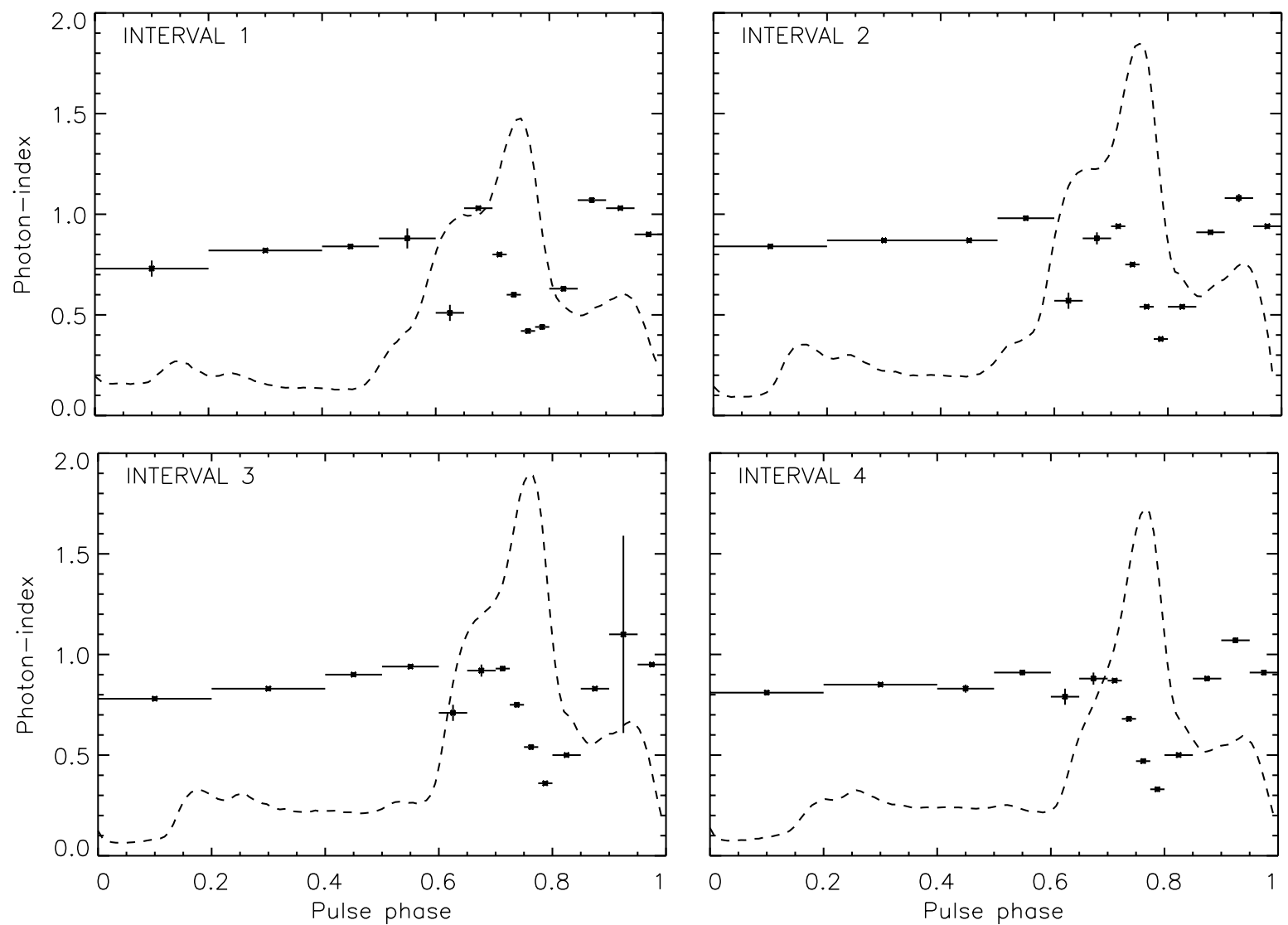

Fig. 6. Photon-index profiles as function of pulse phase for Interval 1(top left), Interval 2 (top right), Interval 3 (bottom left) and Interval 4 (bottom right).

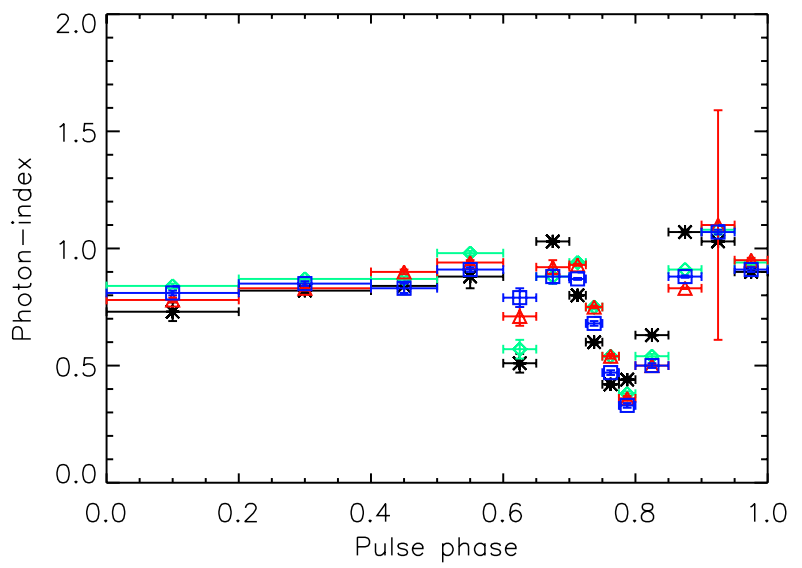

Fig. 7. Photon index $\Gamma$ as function of pulse phase for the four different $35 \mathrm{~d}$ phase intervals (see Table 1): Interval 1 in black (asterisks), Interval 2 in green (diamonds), Interval 3 in red (triangles) and Interval 4 in blue (squares).

all other spectral parameters also tend to show their strongest variability.

\section{Summary and discussion}

We have selected the best data available in the RXTE archive of an observation of a Main-On of Her X-1 to perform detailed pulse phase resolved spectroscopy in the energy range 3.5-75 keV. The observations of November 2002 (cycle no. 323 ) provide the best RXTE coverage of a Main-On (Vasco 2012), such that an attempt could be made to study pulse phase dependent spectral variations also as a function of the phase of the $35 \mathrm{~d}$ modulations - flux and shape of the pulse profiles -, which are believed to be due to precessional motion of the accretion disk and, possibly, the neutron star itself. As spectral model we have used a power law with an exponential cut-off (the highecut model in XSPEC) modified by two line features, a multiplicative cyclotron line (in absorption) and an additive iron fluorescence line (in emission). We have concentrated on three spectral parameters: the centroid energy of the cyclotron line, the photon index $\Gamma$ of the power law component (valid between $3 \mathrm{keV}$ and $\sim 20 \mathrm{keV}$ ), and the intensity of the iron fluorescence line at $6.4 \mathrm{keV}$. For these three parameters, the analysis was performed for four one day long intervals at different mean $35 \mathrm{~d}$ phases $(0.03,0.10,0.15$ and 0.20 , respectively). For the other spectral parameters of the applied spectral model, no 35 day phase dependence was studied.

\subsection{Pulse phase dependence}

The results of the pulse phase resolved analysis can be summarized as follows. We distinguish between results which confirm and improve in statistical significance which was known before and new results.

\subsubsection{Confirmed and improved results}

- All spectral parameters show a strong modulation with pulse phase.

- The strongest variations always appear around the peak of the pulse (at maximum flux). 
D. Vasco et al.: RXTE pulse phase spectroscopy of Her X-1
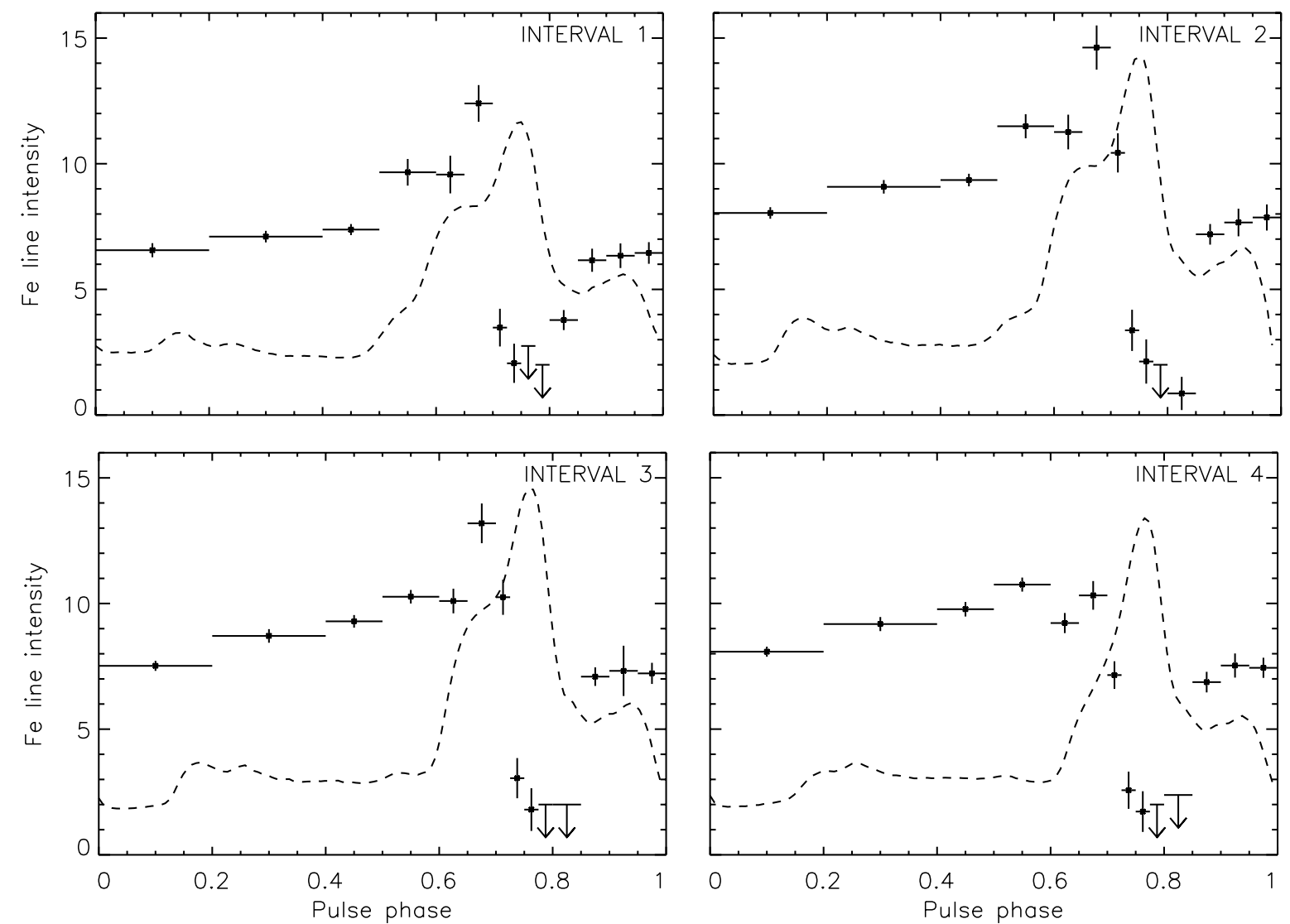

Fig. 8. Iron line intensity profiles as function of pulse phase for Interval 1(top left), Interval 2 (top right), Interval 3 (bottom left) and Interval 4 (bottom right). All intensities less than 1.0 are consistent with zero, we therefore show two sigma upperlimits.

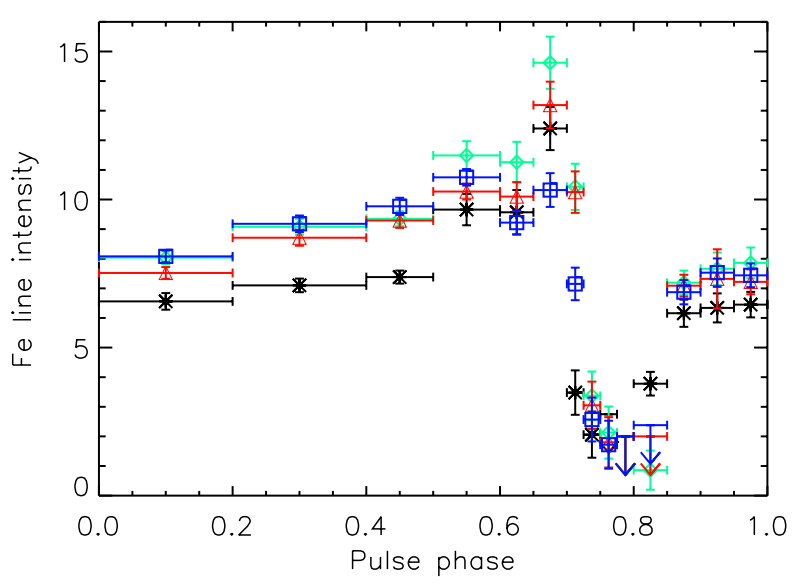

Fig. 9. Iron line intensity profiles for the four different $35 \mathrm{~d}$ phase intervals (see Table 1): Interval 1 in black (asterisks), Interval 2 in green (diamonds), Interval 3 in red (triangles) and Interval 4 in blue (squares).

- The profile of the centroid energy $E_{\text {cyc }}$ of the cyclotron line follows roughly the modulation of the pulse profile (maximum energy around maximum flux). The shape is close to "sinusoidal".

- The photon index profile (Figs. 6 and 7) shows a strong dip close to the pulse peak. The dip around the main peak is a known feature, nicely confirmed here and very well resolved in this analysis.

\subsubsection{New results}

- It is the first time that for Her X-1 a phase profile of the centroid cyclotron line energy $E_{\text {cyc }}$ is produced with such a high resolution and statistical accuracy. The four smallest bins around the peak of the pulse have a width of $1 / 80$ of a phase. The mean variation (minimum to maximum) is $23 \%$ (Figs. 3 and 4). The modulation is close to "sinusoidal"; however, at least two sinusoidal components (with greatly different periods) are needed to model the modulation.

- For 35 d intervals $1-3$, the peak of the $E_{\text {cyc }}$ profile is structured (see Fig. 3), with local maxima near the maximum of the main peak of the pulse profile and around the position of the left shoulder of the main peak. A similar structure is seen in $\tau_{\text {cyc. }}$.

- The folding energy $E_{\text {fold }}$ follows the pulse profile, except for a narrow dip in the peak of the pulse (Fig. 10, upper-right).

- Similarly, the cut-off energy $E_{\text {cut }}$, the width and depth of the cyclotron line $\left(\sigma_{\text {cyc }}\right.$ and $\left.\tau_{\text {cyc }}\right)$, roughly follow the pulse profile, except for higher values at phases $0.0-0.5$ (Fig. 10).

- While the strong dip in the photon index profile (Figs. 6 and 7) close to the main pulse peak was known (even though less well measured), the second, very narrow dip (around phase 0.61 ) is a newly detected feature. We note that this is close to the position of the left shoulder component of the main peak.

- The profile of the intensity of the $6.4 \mathrm{keV}$ iron line show a distinct minimum at maximum flux (Figs. 8 and 9).

- We find that the so called " $10 \mathrm{keV}$ feature" seen in the spectra of several accreting binary pulsars, is around $16 \mathrm{keV}$ in 

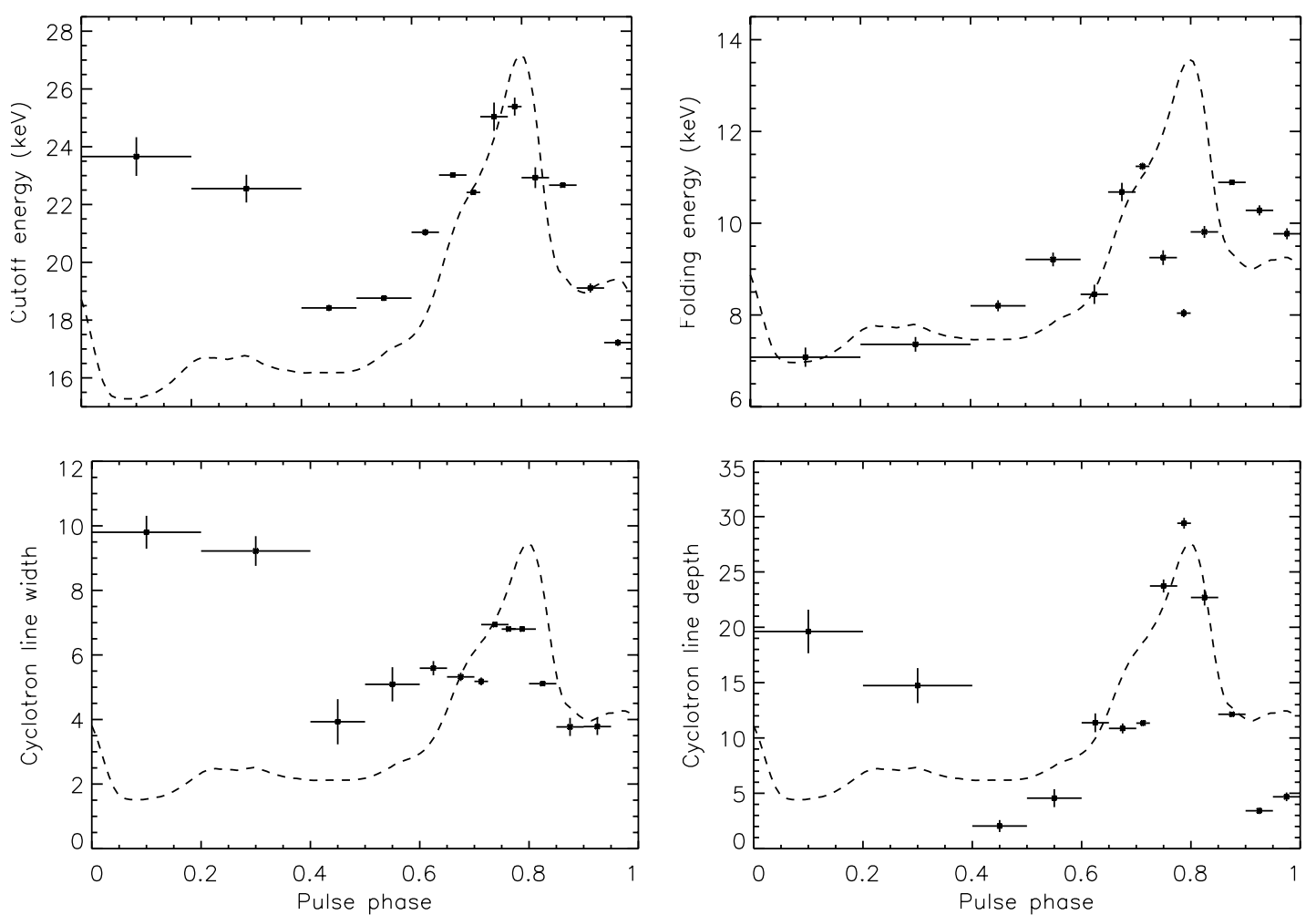

Fig. 10. Pulse phase profiles for the cutoff energy $E_{\text {cut }}$ (top left), the folding energy $E_{\text {fold }}$ (top right), the width of the cyclotron line $\sigma_{\text {cyc }}($ bottom left), and the depth $\tau_{\mathrm{cyc}}$ of the cyclotron line (bottom right) for the total data sets.

Her X-1, with a strong pulse phase dependence: essentially concentrated in the narrow pulse phase interval 0.8-0.9. Spectral fits for all the other phase intervals do not show significant residuals.

\subsection{Precession phase dependence}

With respect to any variation of the spectral parameters (or their pulse phase variations) with $35 \mathrm{~d}$ phase, we consider only the cyclotron line energy $E_{\text {cyc }}$, the photon index $\Gamma$ and the intensity of the iron fluorescence line at $6.4 \mathrm{keV}$. For the other parameters the photon statistics is not sufficient to distinguish between different $35 \mathrm{~d}$ phases. It is the first time that a study of the variation of spectral parameters with $35 \mathrm{~d}$ phase was done. So, the following results are all new.

- The maximum value for $E_{\text {cyc }}$ (reached near the pulse peak) shows a slight, but significant increase with $35 \mathrm{~d}$ phase. The mean value is $41.0 \pm 0.1 \mathrm{keV}$, and the slope is $\sim 0.7 \mathrm{keV}$ per 0.1 units of $35 \mathrm{~d}$ phase.

- The minimum value for $E_{\text {cyc }}$ (around pulse phase 0.3 ) is formally consistent with a constant value of $33.5 \pm 0.5 \mathrm{keV}$ (we would not rule out a small decrease). Together with the maximum increasing, this means that also the peak-to-peak amplitude (mean value $7.6 \pm 0.5 \mathrm{keV}$ ) is increasing with $35 \mathrm{~d}$ phase.

- The shape of the $E_{\text {cyc }}$ profile (see Figs. 4 and 5) is independent of $35 \mathrm{~d}$ phase.

- Similarly, the shape of the $\Gamma$ profiles is independent of $35 \mathrm{~d}$ phase, except for the new narrow dip around pulse phase 0.62 (Figs. 6 and 7), where a trend to a smaller depth from Interval 1 to Interval 4 is seen.
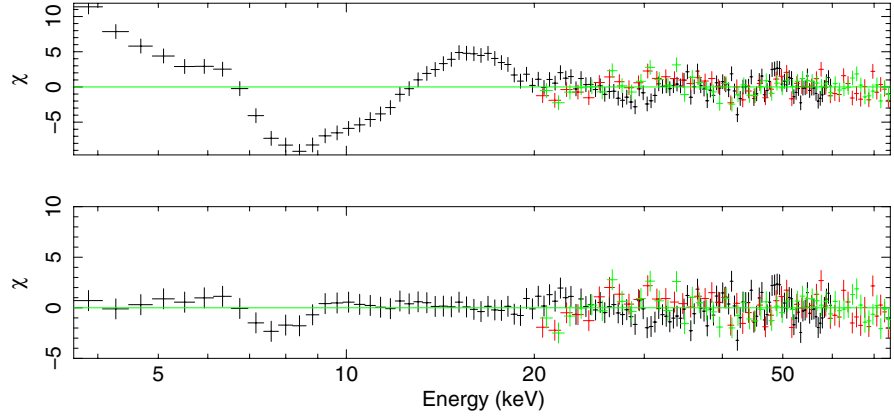

Fig. 11. Residuals of the X-ray spectrum of pulse phase bin $0.80-0.85$ for Interval 3 without (top) and with (bottom) an extra Gaussian component in the fit.

- The shape of the iron line intensity profiles are similar to one another, except for Interval 1 which shows a distinctly lower intensity for pulse phases $<0.6$ (Figs. 8 and 9).

\subsection{Comparison with previous results and physical conclusions}

For Her X-1, the dependence of the spectral parameters on pulse phase have been studied by several authors on the basis of observations with different X-ray instruments (see Introduction). In the following we highlight our new findings and significant improvements and discuss possible physical implications.

Pulse phase dependence of the centroid cyclotron line energy: This modulation is quite common among accreting X-ray pulsars and is generally believed to be due to the changing viewing angle under which the X-ray emitting regions are seen (e.g., 
Kreykenbohm et al. 2004 and references therein). If we adopt the idea, that peaks (high X-ray flux) in the pulse profile can be associated with beamed radiation emitted from the accreting regions around the magnetic poles, then the observed pulse profiles are a representation of the emission characteristics. During a full neutron star rotation we may be seeing different emitting spots (most likely from a complex magnetic field configuration which deviates from a simple dipole (see e.g. Suchy et al. 2008), possibly having a multi-pole configuration) and the emerging beamed radiation under changing angles. The situation is further complicated by gravitational bending. It is therefore not straightforward to interpret observed pulse profiles in terms of accretion geometry and beaming characteristics (see e.g. Caballero et al. 2011). If we, however, assume that the main peak in the pulse profile of Her X-1 is due to a reasonably narrow pencil beam (following e.g., Pravdo et al. 1978; Klochkov et al. 2008), emitted perpendicular to the neutron star surface at the hot spot of the main accreting pole, then we may associate this pulse phase with a situation where the observer is looking down into the accretion column, parallel to the magnetic field lines and the velocity of the in-falling material. At this phase we see the maximum cyclotron line energy and the hardest spectrum (a sharp minimum in photon index $\Gamma$ ) - that is, we are looking deep down to small distances from the neutron star surface where the magnetic field is strongest and the temperature is highest. Here, we also find the largest width and depth of the cyclotron line, as well as the highest $E_{\text {cut }}$ and a local dip within a broad peak of $E_{\text {fold }}$.

Along similar lines, the newly discovered structure of a second (very sharp) minimum in $\Gamma$ around pulse phase 0.61 (Fig. 7), corresponding to the left shoulder of the main peak, may be the signature of a second pole. At this pulse phase we also find indications for another relative maximum in $E_{\text {cyc }}$ (Fig. 3).

Pulse phase dependence of the $6.4 \mathrm{keV}$ iron line flux: Figs. 8 and 9 show a sharp and deep minimum of the intensity of the $6.4 \mathrm{keV}$ iron line, again coincident with the main pulse peak. The profile is very similar to that of $\Gamma$ (Fig. 7) and the Fe line intensity drops to near zero at pulse phase $\sim 0.79$ (where $\Gamma$ is minimal). It is the first time, that such a sharp minimum in the $6.4 \mathrm{keV}$ flux is observed. While Choi et al. (1994), in observations by Ginga, and Zane et al. (2004), in observations by XMM-Newton, had found a sinusoidal-like modulation with a broad minimum around the pulse peak, Oosterbroek et al. (2000), with data from BeppoSAX, were only able to measure the Fe-L line around $1 \mathrm{keV}$, for which they had found a minimum around the pulse peak which was similarly sharp and deep as we now find for the $6.4 \mathrm{keV}$ line. It is generally believed, that the origin for the Fe lines is fluorescence in relatively cool material which is being illuminated by the X-ray beam. For the geometrical site, however, where this fluorescence is taking place, or whether it is predominantly in reflection or in transmission, no general consensus has been reached so far. Several different regions have been proposed (see. e.g. Choi et al. 1994 and references therein): 1) the outer edge of the accretion disk extended in the vertical direction; 2) a corona of the accretion disk; 3) the atmosphere of the companion star; 4) a surface at the Alfvén radius. The Alfvén surface is currently the most popular one. However, also the accretion column was proposed (e.g., Leahy 2001 and İnam \& Baykal 2005), despite the argument by White et al. (1983) that the material in the column may be too hot.

We like to stress the observed fact, that at the pulse phase where we see the highest continuum flux there is essentially no Fe line flux. This should provide some constraints in finding a feasible geometry. If the above adopted scenario is correct, by which we look down into the pencil beam at the pulse peak, the Fe line result may be consistent with a hollow cone geometry, where the majority of the radiation escapes through the empty center of the cone, where there is no material which can fluoresce, while the walls are seen at off-center angles. It is, of course, assumed that the bottom of the cone close to the neutron star surface is filled with X-ray emitting plasma.

The non-dependence of the cyclotron line energy profile on $35 d$ phase and the model of neutron star free precession: one of the results of this study is the finding that the shape of the cyclotron line energy profile ( $E_{\text {cyc }}$ vs. pulse phase) is independent of $35 \mathrm{~d}$ phase (Figs. 3 and 4 ), while the maximum cyclotron line energy increases slightly by $\sim 0.7 \mathrm{keV}$ per 0.1 units of $35 \mathrm{~d}$ phase. If we attribute the strong variations in pulse profile with $35 \mathrm{~d}$ phase to precession of the neutron star, that is to the change of the angle under which we see the X-ray emitting regions, we would expect also the shape of the cyclotron line energy profile to change with $35 \mathrm{~d}$ phase. However, this expectation needs a quantitative analysis. Recently concluded detailed model calculations (Postnov et al. 2012), assuming neutron star free precession, have shown, that under a specific set of assumptions (including a certain multi-pole geometry and emission into narrow pencil beams), the observed pulse profiles in the energy range 9-13 keV (the same as used in Staubert et al. 2010, 2013) can be reproduced with high accuracy. We point out here that the model of Postnov et al. (2012) is an attempt to give a physical explanation of the variation in pulse profiles by neutron star free precession, requiring several specific assumptions, while the template approach of Staubert et al. (2013), mentioned in the Introduction, does not need any physical assumptions, it just makes use of the observed pulse profiles. We plan to investigate whether the here described constancy of the shape of the cyclotron energy profile is consistent with the physical model. In this sense our result opens a new channel to test the model of free precession of the neutron star.

Acknowledgements. This paper is based on observational data taken by the NASA satellite Rossi X-ray Timing Explorer (RXTE). We like to acknowledge the dedication of all people who have contributed to the great success of this mission. D.V. and coauthors thank DLR for financial support through grant 50 OR 0702 and D.K. acknowledges support by the Carl Zeiss Stiftung. We thank the anonymous referee for very valuable comments.

\section{References}

Boynton, P. E., Crosa, L. M., \& Deeter, J. E. 1980, ApJ, 237, 169 Caballero, I., Kraus, U., Santangelo, A., Sasaki, M., \& Kretschmar, P. 2011, A\&A, 526, A131

Choi, C. S., Nagase, F., Makino, F., et al. 1994, ApJ, 437, 449

Coburn, W., Heindl, W. A., Rothschild, R. E., et al. 2002, ApJ, 580, 394

Dal Fiume, D., Orlandini, M., Cusumano, G., et al. 1998, A\&A, 329, L41

Deeter, J. E., Scott, D. M., Boynton, P. E., et al. 1998, ApJ, 502, 802

Endo, T., Nagase, F., \& Mihara, T. 2000, PASJ, 52, 223

Ferrigno, C., Falanga, M., Bozzo, E., et al. 2011, in The X-ray Universe 2011, eds. J.-U. Ness, \& M. Ehle, 69

Gerend, D., \& Boynton, P. E. 1976, ApJ, 209, 562

Giacconi, R., Gursky, H., Kellogg, E., et al. 1973, ApJ, 184, 227

İnam, S. C., \& Baykal, A. 2005, MNRAS, 361, 1393

Kahabka, P. 1987, MPE Rep., 204

Klochkov, D., Shakura, N., Postnov, K., Staubert, R., \& Wilms, J. 2006, Proc. of the 363. WE-Heraeus Seminar on: Neutron Stars and Pulsars (Posters and contributed talks) Physikzentrum Bad Honnef, Germany, May 14-19, eds. W. Becker, \& H. H. Huang, MPE Report, 291, 212

Klochkov, D., Staubert, R., Postnov, K., et al. 2008, A\&A, 482, 907

Kreykenbohm, I., Wilms, J., Coburn, W., et al. 2004, A\&A, 427, 975

Kunz, M., Kendziorra, E., Kretschmar, P., et al. 1996, A\&AS, 120, C233

Leahy, D. A. 2001, in Int. Cosm. Ray Conf., 6, 2536

Lutovinov, A. A., Grebenev, S. A., Pavlinsky, M. N., \& Sunyaev, R. A. 2000, Astron. Lett., 26, 691 
McCray, R. A., Shull, J. M., Boynton, P. E., et al. 1982, ApJ, 262, 301 Oosterbroek, T., Parmar, A. N., Dal Fiume, D., et al. 2000, A\&A, 353, 575 Postnov, K., Shakura, N., \& Staubert, R. 2012, MNRAS, submitted Pravdo, S. H., Bussard, R. W., Becker, R. H., et al. 1978, ApJ, 225, 988 Pravdo, S. H., Bussard, R. W., \& White, N. E. 1979, MNRAS, 188, 5 Reynolds, A. P., Quaintrell, H., Still, M. D., et al. 1997, MNRAS, 288, 43 Rothschild, R. E., Markowitz, A., Rivers, E., et al. 2011, ApJ, 733, 23 Scott, D. M., \& Leahy, D. A. 1999, ApJ, 510, 974

Scott, D. M., Leahy, D. A., \& Wilson, R. B. 2000, ApJ, 539, 392

Soong, Y., Gruber, D. E., Peterson, L. E., \& Rothschild, R. E. 1990, ApJ, 348, 641

Staubert, R., Shakura, N. I., Postnov, K., et al. 2007, A\&A, 465, L25

Staubert, R., Klochkov, D., \& Wilms, J. 2009, A\&A, 500, 883

Staubert, R., Klochkov, D., Vasco, D., \& Wilms, J. 2010, in Proc. of the 8th INTEGRAL Workshop "The Restless Gamma-ray Universe" (INTEGRAL 2010). September 27-30, Dublin Castle, Dublin, Ireland [arXiv: 1110.6717$]$
Staubert, R., Klochkov, D., Vasco, D., et al. 2013, A\&A, in press, DOI: 10.1051/0004-6361/201220316

Suchy, S., Pottschmidt, K., Wilms, J., et al. 2008, ApJ, 675, 1487

Tananbaum, H., Gursky, H., Kellogg, E. M., et al. 1972, ApJ, 174 L143

Trümper, J., Kahabka, P., Oegelman, H., Pietsch, W., \& Voges, W. 1986, ApJ, 300, L63

Trümper, J., Pietsch, W., Reppin, C., et al. 1978, ApJ, 219, L105

Vasco, D. 2012, Ph.D. Thesis, Univ. of Tübingen, Germany

Vasco, D., Klochkov, D., \& Staubert, R. 2011, A\&A, 532, A99

Voges, W., Pietsch, W., Reppin, C., et al. 1982, ApJ, 263, 803

White, N. E., Swank, J. H., \& Holt, S. S. 1983, ApJ, 270, 711

Zane, S., \& Ramsay, G. 2001, in Two Years of Science with Chandra, meeting abstracts from the Symp. held in Washington, DC, 5-7 September

Zane, S., Ramsay, G., Jimenez-Garate, M. A., Willem den Herder, J., \& Hailey, C. J. 2004, MNRAS, 350, 506 